\title{
Anesthetic management of an atrial septal defect in adult-case report
}

\begin{abstract}
The aim of this observation is to discuss the anesthetic management/strategy for percutaneous transcatheter atrial septal defect (ASD) closure in adult patients. Percutaneous occlusion is a commonly performed intervention in ASD closure under transoesophageal (TEE) or intra cardiac echocardiography (ICE) guidance. It is specifically indicated in closure of ostium secundum which represents $60-70 \%$ of the 3 types of ASD inducing a left-to-right shunt and high pulmonary pressure.

General anesthesia or deep sedation is required for most transcatheter cardiac procedures to preventpain, discomfort, untoward hemodynamics and respiratory compromise. There is no consensual protocol for anesthetic management in interventional cardiology. However, preoperative anesthetic evaluation, short-acting medications, possible endotracheal intubation, and peri-procedural monitoring are the determinant security points in such anesthetic management. The objectives in left-to-right shunts are to decrease shunt flow and maintain respiratory and cardiovascular stability. We report a successful anesthesia for percutaneous ASD closure under TEE guidance in a 32 years female patient.
\end{abstract}

Keywords: atrial septal defect closure, anesthetic management, left-to-right shunt
Volume 3 Issue 5 - 2015

\author{
Joseph Koné, I,2 Mohammed Amine El \\ Bouaychi, I,2 Guy Tsala, I,2 Adil Bensouda I,2 \\ Service de Réanimation CCV B- CHU Ibn Sina, Morocco \\ 2Université MohammedV Souissi, Morocco
}

Correspondence: Joseph Kone, MohamedV Souissi, Hopital Ibn Sina, Rabat, Morocco, Tel +212650809760, Email drjosephkone@yahoo.fr

Received: October 07, 2015 | Published: December 14, 2015

\section{Introduction}

Atrial septal defect (ASD) comprises $10 \%$ of congenital cardiac defects in adults. ${ }^{1-4}$ There are three anatomic types of ASD (ostiumprimum, ostium secundum and sinus venosus defect). Ostium secundum is the most common type $(70 \%)^{2,5,6}$ ASD ultimately causes left to right shunt inducing right ventricular distension and hypertrophy, and subsequent pulmonary hypertension from the increased pulmonary artery flow. Defect closure is the curative treatment in this anatomic abnormality. Percutaneous closure is the most common intervention, and is definitively accepted as an effective alternative to sternotomie. ${ }^{7,8}$ In adults, it requires deep sedation (spontaneous breathing) or general anesthesia with tracheal intubation and mechanical ventilation. Distinct anesthetic protocols and perioperative strategies are discussed world-wide in absence of consensus recommendations. The procedure necessitates a preprocedural evaluation, reasonable drug management and periprocedural monitoring of cardio respiratory functions. ${ }^{9-12}$ Aiming to discuss anesthetic possibilities in transcatheter ASD closure, were porta successful general anesthetic for percutaneous ASD closure under TEE guidance in an adult.

\section{Observation}

A 32 years female patient was posted for elective ASD closure under TOE guidance. She had ostium secundum ASD diagnosed seven months prior to the procedure. Her clinical examination revealed moderate breathlessness (NYHA II to III), asthenia and dizziness without chest pain, palpitations or pedal edema. Chest auscultation revealed a $2 / 6$ systolic and diastolic murmur in the pulmonary area and split second heart sound. The rest of the physical examination was normal. ECG showed an incomplete right bundle branch block, a PR interval of 0.20 second, and bi-atrial hypertrophy. Transthoracic echocardiography confirmed dilatation of both atria and revealed a $24 \mathrm{~mm}$ ostium secundum ASD, with a left-to-right shunt, mild pulmonary hypertension (pulmonary arterial systolic pressure of 40
$\mathrm{mmHg}$ ) and a left ventricular ejection fraction of $62 \%$. Pre-anesthetic evaluation suggested an ASA III status based on grade III NYHA functional classification (important limitation of activity, even during light duties: e.g. walking short distances from 20 to $100 \mathrm{~m}$ ). Her airway was Mallampati I; blood pressure and heart rate remained normal.

\section{Anesthetic management}

In operating theater a peripheral intravenous line (IV) was inserted using an 18 Gauge cannula. Antibiotic prophylaxis (cefuroxime $1,5 \mathrm{~g}$ ), heparin $100 \mu \mathrm{g} / \mathrm{kg}$ and normal saline solution infusion were given. During anesthesia, hemodynamic and respiratory parameters (noninvasive blood pressure, heart rate, ECG, SpO2and EtCO) were monitored. Following 3 minutes pre-oxygenation, general anesthesia was induced by IV bolus of propofol $160 \mathrm{mg}$, fentanyl $150 \mu \mathrm{g}$ and rocuronium $40 \mathrm{mg}$. Patient was then orally intubated with a $7.0 \mathrm{~mm}$ cuffed tracheal tube for controlled mechanical ventilation. Anesthesia was maintained with propofol $6 \mathrm{mg} / \mathrm{kg} / \mathrm{hdelivered} \mathrm{via} \mathrm{syringe} \mathrm{infusion}$ pump. Before femoral venous catheterization, local infiltration was performed with $8 \mathrm{ml}$ of $1 \%$ lidocaine.

Intra procedural monitoring was normal, aside from one episode of bradycardia resolved with IV atropine $500 \mu \mathrm{g}$. The ASD closure was completed without complications, lasted 76 minutes, and consisted of amplatzer occlusion technique after a balloon test under TEE guidance Figure 1(A \& B). Propofol infusion was then stopped and neostigmine administered for the reversal of neuromuscular blockade. Patient was extubated and monitored in recovery room using Aldrete scale based surveillance, then transferred into the cardiac intensive unit. Post procedural analgesia paracetamol $1000 \mathrm{mg}$ every six hours and acetyl salicylate DL lysine $160 \mathrm{mg}$ daily as antithrombotic. She was discharged the following day.

\section{Discussion}

We report a case of general anesthesia in a patient who underwent an ostium secundum ASD closure by an amplatzer with TEE doppler 
guidance. There are three types of ASD: sinus venosus, ostiumprimum, ostium secundum ASD, (which accounts for 70\%). Typically, the fossa ovalis is involved in the mid septallocation in most cases of ASD. ${ }^{5}$ Some unusual presentations have been described. ${ }^{13}$ Significant or complex ASD is often associated with other anatomical anomalies such as Eisenmenger or Lutembacher syndromes. ${ }^{14,15}$ Patients with congenital heart disease can remain asymptomatic until adulthood age., ${ }^{416}$ Our patient was 32 years old, and underwent previously 3 normal pregnancies without any remarkable cardiac dysfunction.
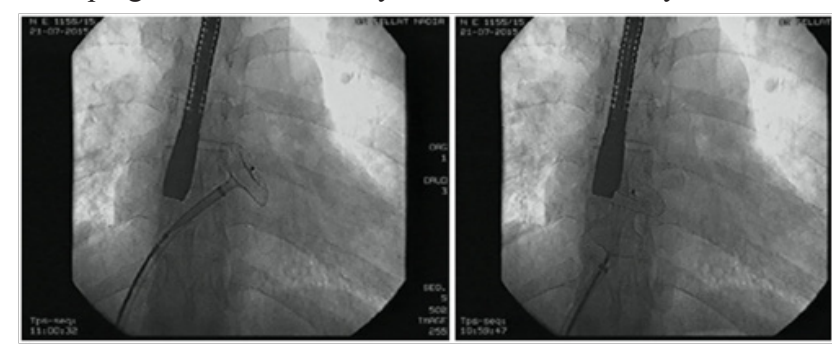

Figure I (A \& B) Closure device in place.

Anesthetic management must consider major complications like pulmonary arterial hypertension, right heart dysfunction and the severity of shunt. The diagnosing the magnitude of these complications are evaluated by doppler echocardiography. ${ }^{17}$

Despite the fact that many protocols have been reported for cardiac catheterization, controversies between deep sedation with spontaneous breathing and general anesthesia with controlled ventilation remain. There is no consensual recommendation for in-operative anesthetic drugs..$^{10,12,18,19}$

We performed general anesthesia with controlled ventilation to ensure more complete hemodynamic and respiratory control. Physiological parameters were adjusted to avoid disturbance in gas exchange and to prevent an acute shunt reversal or an increase in the pulmonary vascular resistance with development of pulmonary arterial hypertension. Challenges are to prevent air embolism, maintain adequate preload, optimize cardiac contractility normalize heart and respiratory rates while being vigilant for atrial arrhythmias.

Propofol is a frequently used hypnotic agent in international cardiology, due to its short duration. But cardiovascular depression associated with its usage has been described. In many studies, propofol and ketamine, in combination have been employed ASD closure. Advantages of that combination are the short-action duration of propofol, the analgesic effect of ketamine in addition to its cardiovascular stability. Studies utilizing etomidate, remifentanil, midazolam or sevoflurane are listed in transcatheter cardiac procedures with success in regards to their manageability and absence of major cardiac side effects. ${ }^{19}$ Emesis is not a rare event in TEE procedures, and tracheal intubation provides some protection against gastric acid pulmonary aspiration. Perioperative antibiotic was to prevent subacute bacterial endocarditis and lysine acetyl salicylate for antiplatelet action.

Anesthetic management for ASD closure in adult patients should avoid undesirable cardiovascular events such as increase in pulmonary vascular resistance, increase of blood flow through the shunt, hypotension, tachycardia, hypoxia and air embolism. Shortacting hypnotics are preferable, and preventing air embolism and pulmonary aspiration of gastric acid is a safety measure.

\section{Acknowledgments}

None.

\section{Conflicts of Interset}

None.

\section{References}

1. Roy R, Prasad A. Anaesthesia Management of a Patient with Large Atrial Septal Defect with Moderate Pulmonary Hypertension for Total Abdominal Hysterectomy. J Anesth Crit Care Open Access. 2015;2(6):00075.

2. Bedford $D$. The anatomical types of atrial septal defect: Their incidence and clinical diagnosis. Am J Cardiol. 1960;6:568-574.

3. Campbell M. Natural history of atrial septal defects. Br Heart J. 1970;32(6):820-826.

4. Mulder BJ. Epidemiology of adult congenital heart disease: demographic variations worldwide. Neth Heart J. 2012;20(12):505-508.

5. Brickner ME, Hillis LD, Lange RA. Congenital heart disease in adults N Engl J Med. 2000;342(4):256-263.

6. Feldt RH, Avasthey P, Yoshimasu F, et al. Incidence of congenital heart disease in children born to residents of Olmsted County, Minnesota, 1950-1969. Mayo Clin Proc. 1971;46(12):794-799.

7. Du Z, Hijazi Z, Kleinman C, et al. Comparison between transcatheter and surgical closure of secundum atrial septal defect in children and adults: Results of a multicenter nonrandomized trial. J Am Coll Cardiol. 2002;39(11):1836-1844.

8. Guo JJ, Luo YK, Chen ZY. Long-termout comes of device closure of very large secundum atrial septal defects: a comparison of transcathetervs intra operative approaches. Clin Cardiol. 2012;35(10):626-631.

9. Choi YS, Kwak YL, Jeon DH, et al. Anesthesia for Robotic Repair of the Atrial Septal Defect - A case report. Korean J Anesthesiol. 2007;52(3): $371-375$.

10. Mehmet E, Gökhan Ö, AyhanKılıç, et al. Anesthesia Management for Transcatheter Atrial Septal Defect Closure in Pediatric Patients. Journal of Clinical and Analytical Medicine. 2015; 1-3.

11. Karagöz AH, Ankay-YılbaşA, Kanbak M, et al. Anesthesia for percutaneous transcatheter closure of atrial and ventricular septal defects in pediatric patients. Turk J Pediatr. 2013;55(6):628-632.

12. Calvert PA, Klein AA. Anaesthesia for percutaneous closure of atrial septal defects. Contin Educ Anaesth Crit Care Pain. 2008;8(1):16-20.

13. Lee MG, Ko JS, Yoon HJ, et al. An unusual presentation of an atrial septal defect. J Cardio vasc Ultrasound. 2009;17(4):151-152.

14. Pascual I, Sucarrats SG, Clavel L, et al. Eisenmenger's Syndrome in Context of a Complete Atrioventricular Canal Defect. J Pediatr Neonatal Care. 2014;1(6):00042.

15. Kulkarni SS, Sakaria AK, Mahajan SK, et al. Lutembacher's syndrome $J$ Cardiovasc Dis Res. 2012;3(2):179-181.

16. Zomer AC, Vaartjes I, Grobbee DE, et al. Adult congenital heart disease: new challenges. Int J Cardiol. 2013;163(2):105-107.

17. Hernández F, García-Tejada J, Velázquez M. Intracardiac ; Echocardiography and Percutaneous Closure of Atrial Septal Defects in Adults. Rev Esp Cardiol. 2008;61:465-470.

18. Chari VRR, Suryawanshi CM, Sahu P. Anesthetic management of a case of a large atrial septal defect with mild pulmonary hypertension for emergency cesarean section. Anaesth Pain \& Intensive Care. 2013;17(2):182-184.

19. Hanslik A, Moysich A, Laser KT, et al. Percutaneous closure of atrial septal defects in spontaneously breathing children under deep sedation: a feasible and safe concept. Pediatr Cardiol. 2014;35(2):215-222. 